\title{
ARID1A gene knockdown promotes neuroblastoma migration and invasion
}

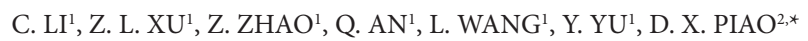 \\ ${ }^{1}$ Department of Pediatric Surgery, The First Affiliated Hospital of Harbin Medical University, Harbin 150081, People's Republic of China; \\ ${ }^{2}$ Department of Colorectal Surgery, The First Affiliated Hospital of Harbin Medical University, Harbin 150081, People's Republic of China \\ ${ }^{*}$ Correspondence: daxun_piao@163.com
}

Received July 6, 2016 / Accepted November 6, 2016

\begin{abstract}
Neuroblastoma is the most common extracranial solid tumor in childhood which often acquires drug resistance and becomes aggressive phenotypes. The high-risk patients suffer from high mortality due to the limitation of the treatment strategies. ARID1A (AT-rich interactive domain-containing protein 1A), a subunit of SWI/SNF complexes, is considered as a tumor suppressor in many cancers. The aim of the present study was to investigate the effect of ARID1A on migration and invasion in neuroblastoma cells. The shRNA targeting ARID1A was designed and delivered into SK-N-SH cells to knock down ARID1A expression. Knockdown of ARID1A by shRNA significantly increased the viability and invasion ability, and caused G1 arrest inhibition and DNA synthesis increase in SK-N-SH cells. Moreover, Knockdown of ARID1A increased the activity and expression of matrix metalloproteinase (MMP)-2 and -9 in SK-N-SH cells. Furthermore, ARID1A knockdown caused diminished expression of E-cadherin, enhanced expression of $\mathrm{N}$-cadherin and $\beta$-catenin nuclear translocation in SK-N-SH cells. These results suggest that loss of ARID1A may associate with the promotion of invasion and metastasis of neuroblastoma. Our findings indicate ARID1A is a tumor suppressor in neuroblastoma.
\end{abstract}

Key words: neuroblastoma, ARID1A, shRNA, SH-N-SH cell, metastasis

Neuroblastoma, an embryonal tumor in the autonomic nervous system, is the most common extracranial solid tumor in childhood $[1,2]$. It characterized by broad spectrum of clinical behavior and accounts for approximate $7 \%$ of malignancies in children. Even worse, more than half of the neuroblastoma patients have metastatic disease [2-4]. In addition, neuroblastoma often acquires drug resistance to chemotherapeutic drugs and becomes aggressive phenotypes. Therefore, although a number of basic and clinical studies focused on the development of the treatment strategies and most patients achieved remission, the high-risk patients still suffer from high ratio of mortality. Thus, a better understanding of the mechanisms involved in the development of neuroblastoma would be beneficial to improve the treatment for this disease.

Growing evidence has indicated that dysregulation of ATP-dependent chromatin remodeling complexes was involved in a variety of cancers $[5,6]$. SWitch/Sucrose NonFermentable (SWI/SNF), one of ATP-dependent chromatin remodelers, is the most commonly dysregulated in cancer. AT-rich interactive domain-containing protein $1 \mathrm{~A}$ (ARID1A), a subunit of SWI/SNF complexes, is the most frequently mutated gene in cancer [7]. ARID1A has been considered as a tumor suppressor through the discoveries of inactivating ARID1A mutations in various cancers and the inverse correlation between ARID1A expression and tumor stage [7-9]. In a clinical study of neuroblastoma, Mark Sausen et al. found that genetic alterations of ARID1A was associated with early treatment failure and decreased survival [10], which indicated that ARID1A mutation contributed to the development of neuroblastoma. However, little is known about the function or mechanisms of ARID1A in neuroblastoma. The present study aims to clarify the role of ARID1A in proliferation, migration and invasion in neuroblastoma cell line SK-N-SH.

\section{Materials and methods}

SK-N-SH cells culture and transient transfection. The human neuroblastoma cell line SK-N-SH was purchased from Cell Bank of Chinese Academy of Sciences (Shanghai, China). The cells were cultured in Dulbecco's modified Eagle medium (DMEM) (Invitrogen, Carlsbad, CA, USA) supplemented with $10 \%$ fetal bovine serum (FBS) (Hyclone, Logan, UT, USA) and 
antibiotics (100 U/ml penicillin and $100 \mu \mathrm{g} / \mathrm{ml}$ streptomycin) at $37^{\circ} \mathrm{C}$ in a humidified $5 \% \mathrm{CO}_{2}$ atmosphere. The cells in passage 3-5 were used for transient transfection.

Plasmid and stable transfection. A shRNA target to ARID1A (1: 5'-GCCAAGGAGAGCAGAGTAA-3', 2: 5'-GCCCTAACATGGCCAATAT-3') and a control scramble sequence (shCon) (5'-GATCCCCTTCTCCGAACGTGTCACGTTTCAAGAGAACGTGACACGTTCGGAGAATTTTT-3') were synthesized by and purchased from Genscript (Nanjing, China) and inserted into the BamHI/ HindIII site of linearized pRNA-H1.1 vector to yield the pRNA-H1.1-shRNA construct. The cells were transfected with pRNA-H1.1-shARID1A or pRNA-H1.1-shCon plasmid at the concentration of $1 \mu \mathrm{g} / \mathrm{ml}$. Lipofectamine 2000 (Invitrogen, Carlsbad, CA, USA) was used as the transfection reagent. Stable SK-N-SH cells containing the pRNA-H1.1-shRNA was established after selection with $400 \mu \mathrm{g} / \mathrm{ml} \mathrm{G} 418$ (Invitrogen) for 2 weeks. The efficiency of the transfection was examined using immunofluorescence staining, and realtime PCR and Western blotting.

MTT assay. The cells were dispensed into 96-well plates at $3 \times 10^{3} /$ well. After being cultured for indicated periods, $100 \mu$ l of MTT (final concentration: $5 \mathrm{mg} / \mathrm{ml}$ ) was added and incubated for $4 \mathrm{~h}$. Subsequently, the entire supernatant was discarded and $200 \mu$ of dimethyl sulfoxide (DMSO) was added into each well. After being incubated at $37^{\circ} \mathrm{C}$ for $10 \mathrm{~min}$, the absorbance at $490 \mathrm{~nm}$ of each well was determined using an ELISA reader (ELX-800, Bio-Tek Instruments, Winooski, VT, USA).

Cell cycle analysis. The cells were cultured in 6-well plates for $24 \mathrm{~h}$ or $48 \mathrm{~h}$. The cells were trypsinized, and counted cells were harvested, fixed in pre-cold $70 \%$ ethanol at $4{ }^{\circ} \mathrm{C}$ for $2 \mathrm{~h}$. Following incubation with $25 \mu \mathrm{l}$ propidium iodide (PI) and $10 \mu \mathrm{l}$ RNase A for 30 min in the dark at $37^{\circ} \mathrm{C}$, the cells were analyzed by flow cytometry (C6, BD Biosciences, San Jose, CA, USA).

Colony formation assay. The cells were cultured in 35$\mathrm{mm}$ dishes at 100 cells/dish for 1 week and fixed using $4 \%$ paraformaldehyde (PFA) for $20 \mathrm{~min}$ at room temperature. The colonies were stained with Wright-Giemsa Stain solution

Table 1. Primary antibodies

\begin{tabular}{lllll}
\hline Name & applications & Dilution & Cat. No. & Company \\
\hline ARID1A & IF, WB & $1: 300,1: 1000$ & ab182560 & Abcam \\
Ki67 & IF & $1: 200$ & sc-25280 & CST \\
E-cadherin & IF, WB & $1: 300,1: 400$ & BA0474 & Boster \\
N-cadherin & IF, WB & $1: 300,1: 400$ & BA0473 & Boster \\
Vimentin & IF, WB & $1: 200,1: 500$ & bs-8533R & Bioss \\
$\beta$-catenin & IF, WB & $1: 300,1: 400$ & BA0426 & Boster \\
MMP2 & WB & $1: 400$ & BA0569 & Boster \\
MMP9 & WB & $1: 400$ & BA0573 & Boster \\
Histone H3 & WB & $1: 400$ & bs-17422R & Bioss \\
$\beta$-actin & WB & $1: 1000$ & sc-47778 & Santa cruz \\
\hline
\end{tabular}

Abcam, Cambridge, UK; Santa Cruz Biotechnology. CST (Cell Signaling Technology), Danvers, MA, USA; Santa Cruz, CA, USA; Boster, Wuhan, China; Bioss, Beijing, China.
(Nanjing Jiancheng Bioengineering Institute, Nanjing, China). Cell colonies were counted and captured under a microscope (AE31, Motic China Group Co. Ltd., Xiamen, China).

Wound-healing assay. Cell migration was determined using the wound-healing assay. The cells were cultured in 6-well plates to confluency. Cells were cultured in serum-free medium supplemented with mitomycin $1 \mu \mathrm{g} / \mathrm{ml}$ for $1 \mathrm{~h}$. Subsequently, a sterile $200 \mu$ pipette tip was used to create a line-shaped scratch at the surface of the monolayer. Cells were washed using serum-free DMEM to remove the detaching cells and cultured in serum-free DMEM. The degree of wound healing was observed and images were captured at 0,12 and $24 \mathrm{~h}$. The cell migration rate was calculated as following: migration rate $(\%)=(1-$ wound distance/initial distance $) \times 100 \%$.

Transwell invasion assay. The invasion ability of cells was evaluated using Transwell $(8 \mu \mathrm{m}$ pore size, Corning Incorporated, Corning, NY, USA). The upper surface of the filter was coated with Matrigel (BD Biosciences) at $37^{\circ} \mathrm{C}$ for $2 \mathrm{~h}$. The $2 \times 10^{4}$ cells in $200 \mu \mathrm{l}$ of serum-free DMEM were seeded into the upper chamber of the Transwell insert, and the lower chamber was filled with $800 \mu \mathrm{l}$ DMEM with $20 \%$ FBS. Following $24 \mathrm{~h}$ of incubation, the invasive cells on the bottom side of the membrane were fixed using 4\% PFA for $20 \mathrm{~min}$ and staining using $0.5 \%$ crystal violet for $5 \mathrm{~min}$. Finally, the number of invasive cells was counted in 5 random fields under a microscope (AE31, magnification, $200 \times$ ).

Immunofluorescence staining. Cells were cultured on glass coverslips were washed using PBS and fixed using 4\% PFA for $15 \mathrm{~min}$ at room temperature. The cells were permeabilized with $0.1 \%$ triton X-100 (Amresco, solon, OH, USA) for $30 \mathrm{~min}$ at room temperature. After a wash stage using PBS, the cells were blocked using goat serum (Solarbio Science \& Technology, Beijing, China) for $15 \mathrm{~min}$ and incubated with the primary antibodies at $4{ }^{\circ} \mathrm{C}$ overnight. The cells were then washed with PBS and incubated with Cy3 labeled anti-goat or mouse IgG (1:200, Beyotime Institute of Biotechnology, Haimen, China) for $1 \mathrm{~h}$ at room temperature. After washing, cells were counterstained with 4'6-diamidino-2-phenylindole (DAPI) and observed using a fluorescence microscope (BX53, Olympus, Tokyo, Japan).

MMP Gelatin Zymography. Gelatin zymography was used to quantify MMP-2 and 9 enzymatic activities in vitro. The culture media were collected and centrifuged at $1,000 \mathrm{~g}$ for 10 $\min$ at $4{ }^{\circ} \mathrm{C}$ to remove cells and debris. The media samples were loaded on to a $10 \%$ SDSPAGE copolymerized with $1 \mathrm{mg} / \mathrm{ml}$ gelatin (Sigma-Aldrich, St Louis, MO, USA) and electrophoretically separated at $70 \mathrm{~V}$ for $2 \mathrm{~h}$. The gel was then washed with a eluate buffer (containing 2.5\% Triton X-100, 50 mM Tris-HCl, $\left.5 \mathrm{mM} \mathrm{CaCl}_{2}, 1 \mu \mathrm{M} \mathrm{ZnCl}_{2}, \mathrm{pH} 7.6\right)$ to remove SDS and incubated in a developing buffer (containing $50 \mathrm{mM}$ Tris- $\mathrm{HCl}, 5 \mathrm{mM}$ $\left.\mathrm{CaCl}_{2}, 1 \mu \mathrm{M} \mathrm{ZnCl}_{2}, 0.02 \% \mathrm{Brij}, 0.2 \mathrm{M} \mathrm{NaCl}\right) 37^{\circ} \mathrm{C}$ for $40 \mathrm{~h}$. After the incubation, the gel was stained in $30 \%$ methanol, $10 \%$ acetic acid, and $0.05 \%$ Coomassie brilliant blue (w/v) for $3 \mathrm{~h}$ and de-stained in de-staining solution. MMP- 2 and 9 activities were quantified by analyzing band densities using Image J software (National Institutes of Health, Bethesda, MD, USA). 
RNA isolation and real time PCR. Total RNA was extracted from the cells using a RNA Extraction Kit (BioTeke, Beijing, China). The cDNA was reversely transcribed with oligonucleotide primer using super M-MLV (BioTeke, Beijing, China). Quantitative real time PCR was performed on $1 \mu \mathrm{l}$ cDNA using the $2 \times$ Power Taq PCR Master Mix (BioTeke) and SYBR Green (Solarbio) on an Exicycler 96 (Bioneer, Daejeon, Korea). $\beta$-actin was used as a loading control. Relative gene expression levels were calculated using the $2^{-\Delta \Delta C t}$ method. The sequences of primers are as follows: ARID1A forward: 5'-GGACCTCTATCGCCTCTATGTG-3', reverse: 5'-GGACTTCTTGGAATCAGCAG-3'; $\beta$-actin, forward: 5'CTTAGTTGCGTTACACCCTTTCTTG-3', reverse: 5'CTGTCACCTTCACCGTTCCAGTTT-3'.

Western blotting analysis. The cell sample was homogenized in a RIPA lysis buffer (Beyotime, Nantong, China) containing $1 \mathrm{mM}$ phenylmethanesulfonyl fluoride on ice. The protein concentration was determined using a BCA protein assay kit (Beyotime, Nantong, China). The protein samples that boiled with loading buffer were subjected to SDS-PAGE using $10 \%$ gels. The separated proteins were transferred to polyvinylidene difuoride membranes and blocked in 5\% non fat milk in Tris buffered saline with tween-20 (TBST) for 1h. Afterward, the membrane was incubated with primary antibodies overnight at $4{ }^{\circ} \mathrm{C}$ and incubated with anti-rabbit IgG-horseradish peroxidase -conjugated secondary antibodies (1:5000, Beyotime) at $37^{\circ} \mathrm{C}$ for $45 \mathrm{~min}$. At last, protein bands were visualized by the enhanced chemiluminescence (ECL) regent (Wanleibio, Shenyang, China).

Statistical analysis. Statistical analysis was performed using SPSS 19.0 for windows (IBM, New York, NY, USA). Data were analyzed by one-way analysis of variance (ANOVA) followed by the Fisher's least significant difference (LSD) test for post hoc comparisons. $\mathrm{P}<0.05$ was considered statistically significant.

\section{Results}

Efficiency of shRNA-mediated knockdown of ARID1A. In order to investigate the function of ARID1A in cancer, we selected the SK-N-SH cell line, which expresses the highest level of ARID1A (Figure 1A). To determine the effects of shRNA treatment on ARID1A expression, the ARID1A mRNA
A
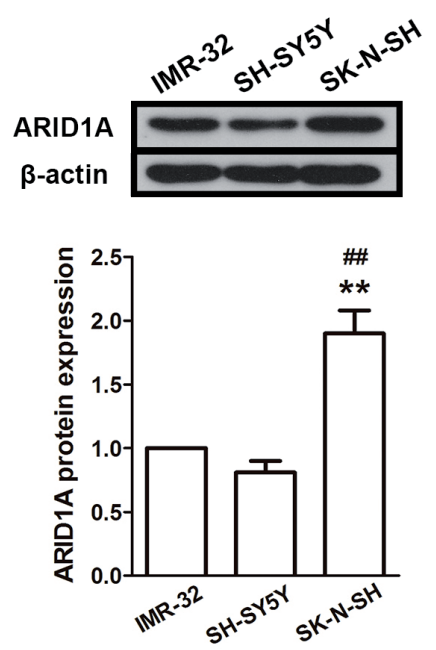

D

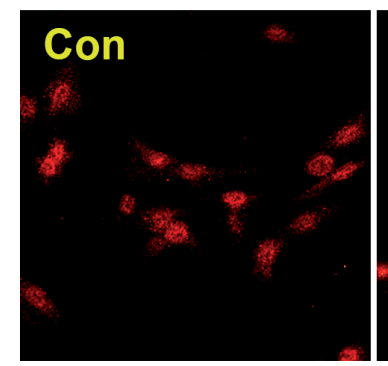

B

ARID1A mRNA

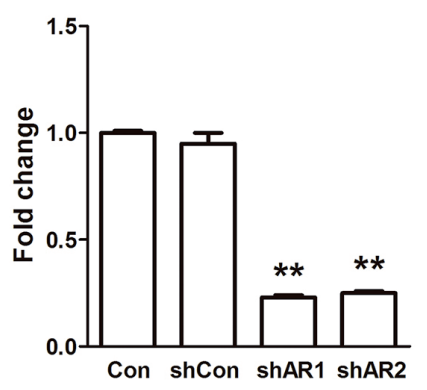

C
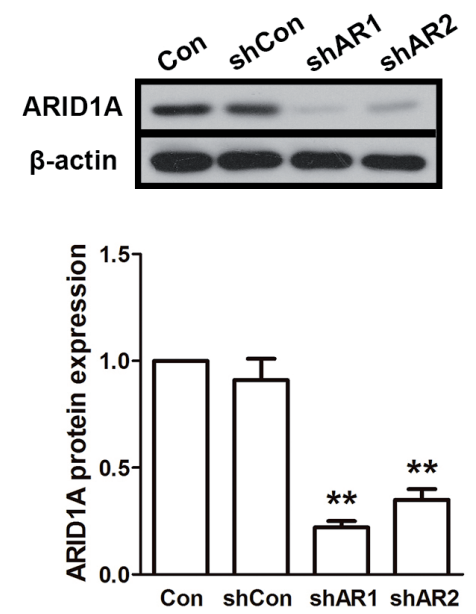

Figure 1. Evaluation of ARID1A knockdown. (A) Protein expression of ARID1A in different neuroblastoma cell lines. The transfection efficiency of shARID1A was examined using real-time PCR (B), Western blotting (C) and immunofluorescence staining (D). Transfection of shRNA plasmid against ARID1A induced significant reduction of ARID1A $\mathbf{m R N A}$ and protein expression. Scale bar: $50 \mu \mathrm{m}$. Values are expressed as mean \pm standard deviation ( $\mathrm{n}=3$ ). ${ }^{\star *} P<0.01$ vs. shCon. Con: Control SK-N-SH cells; shCon: SK-N-SH cells transfected with scramble shRNA; shAR: SK-N-SH cells transfected with shRNA against ARID1A. 
A

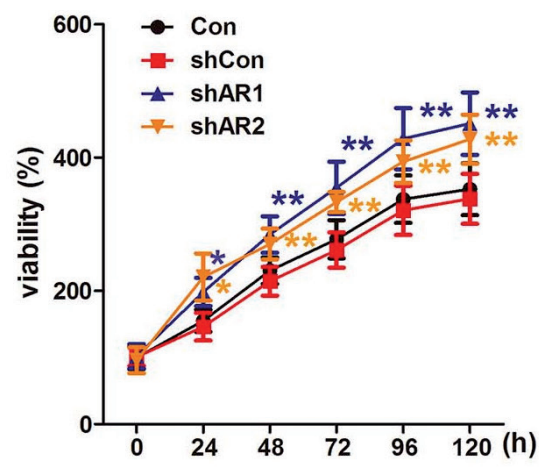

B
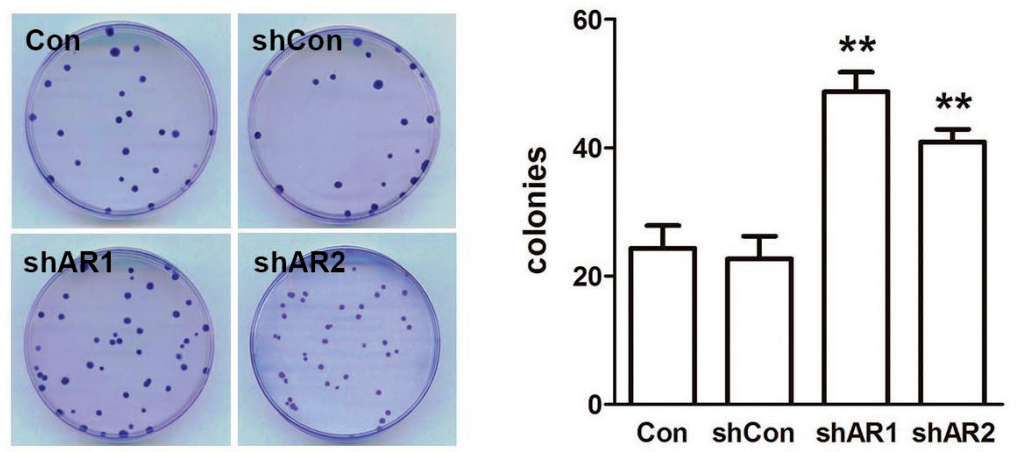

C

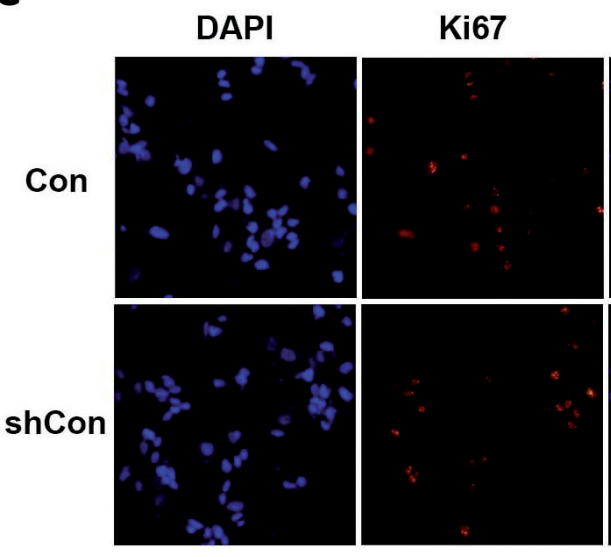

Merge
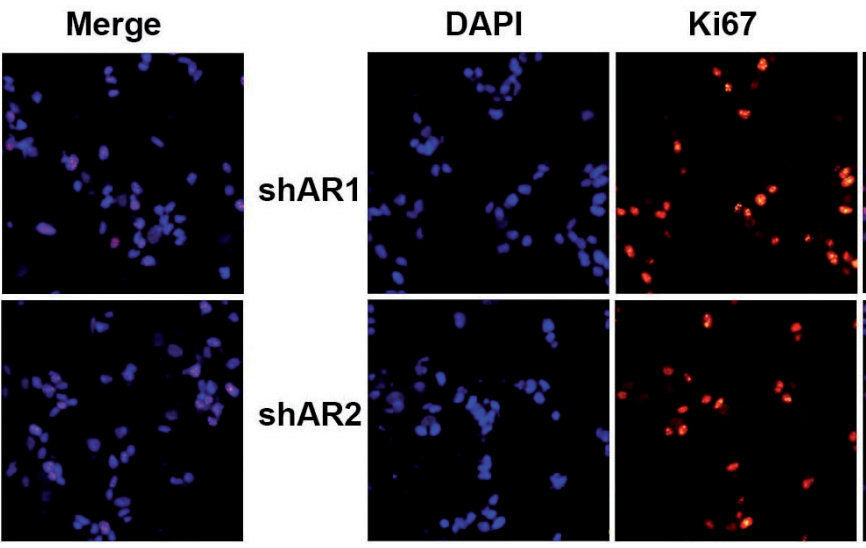

Merge

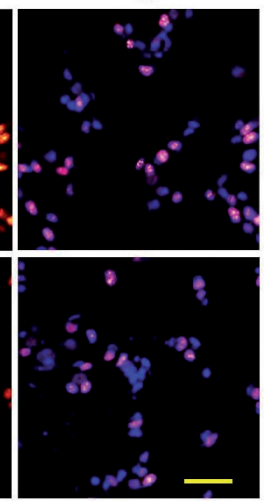

Figure 2. Knockdown of ARID1A promotes in vitro cell proliferation and colony formation in SK-N-SH cells. SK-N-SH cell growth was tested by MTT (A), colony formation assay (B) and Ki67 immunofluorescence staining (C). ARID1A knockdown caused increased cell growth in SK-N-SH cells. Scale bar: $50 \mu \mathrm{m}$. Values are expressed as mean \pm standard deviation $(n=3) .{ }^{* *} P<0.01$ vs. shCon. Con: Control SK-N-SH cells; shCon: SK-N-SH cells transfected with scramble shRNA; shAR: SK-N-SH cells transfected with shRNA against ARID1A.

A

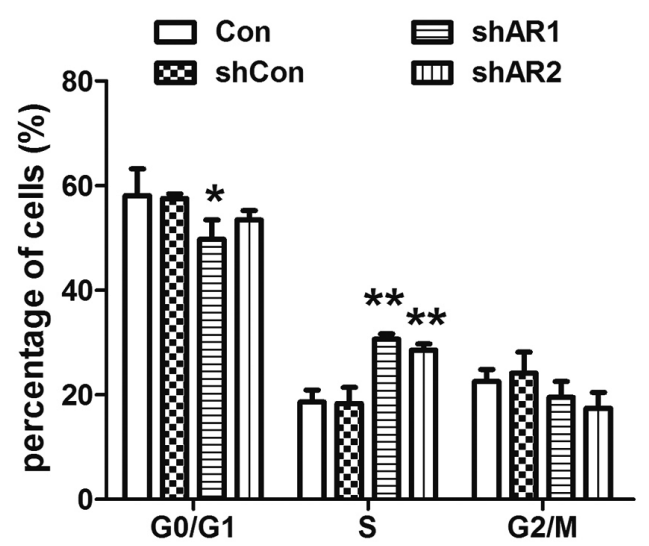

B

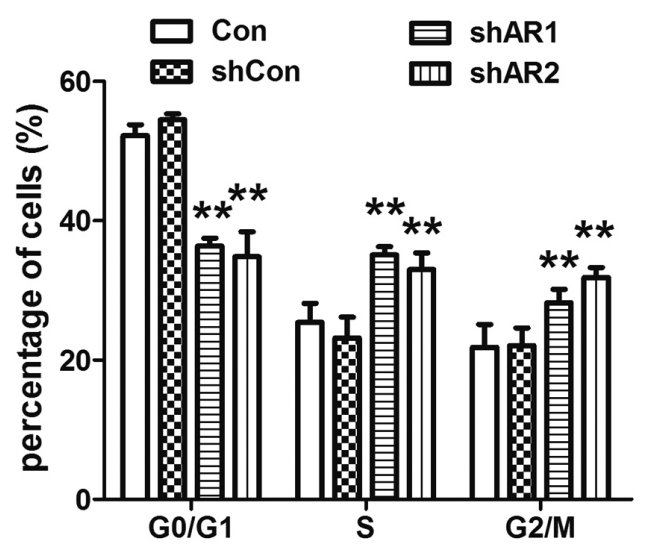

Figure 3. ARID1A knockdown affects cell cycles in SK-N-SH cells. The cell cycle of SK-N-SH cells was examined using low cytometry at $24 \mathrm{~h}$ (A) and 48h (B). shARID1A induced decreased percentage of cells in G0/G1 phase and increased percentage of cells in S and G2/M phase. Values are expressed as mean \pm standard deviation $(n=3) .{ }^{*} P<0.01$ vs. shCon. Con: Control SK-N-SH cells; shCon: SK-N-SH cells transfected with scramble shRNA; shAR: SK-N-SH cells transfected with shRNA against ARID1A. 
and protein expression levels were analyzed by real-time PCR, Western blot and immunofluorescence staining, respectively. Compared with scramble shRNA, shARID1A transfection significantly reduced ARID1A mRNA and protein expression levels in SK-N-SH cells (Figure 1B, 1C). Immunofluorescence staining also reflected the diminished expression of ARID1A in SK-N-SH cells induced by shARID1A (Figure 1D). These results indicate the success of the shRNA-mediated ARID1A knockdown.

ARID1A knockdown promotes SK-N-SH cell growth. The effects of ARID1A knockdown on SK-N-SH cell growth were evaluated by MTT, colony formation assay and immunofluorescence staining of Ki67. As shown in Figure 2A, cells with ARID1A knockdown proliferated markedly faster than cells transfected with scrambled shRNA during the first 120 $\mathrm{h}$ after the cells were seeded. Similarly, Ki67 expression was also dramatically increased in ARID1A knockdown SK-N-SH cells (Figure 2C). Additionally, colony formation assay showed that ARID1A knockdown significantly enhanced the colony formation ability (Figure 2B). The results suggest that ARID1A knockdown promotes SK-N-SH cell growth.

Knockdown of ARID1A alters the cell cycle in SK-N$\mathrm{SH}$ cells. Flow cytometry assay was used to determine the effect of ARID1A knockdown on the cell cycle of SK-N-SH cells. As illustrated in Figure 3, after being cultured for $24 \mathrm{~h}$, one of the shARID1A caused significantly decreased cell percentage of G0/G1 ( $P<0.01$ vs. shCon), the other one also decreased the cells in G0/G1 phase, but did not

A
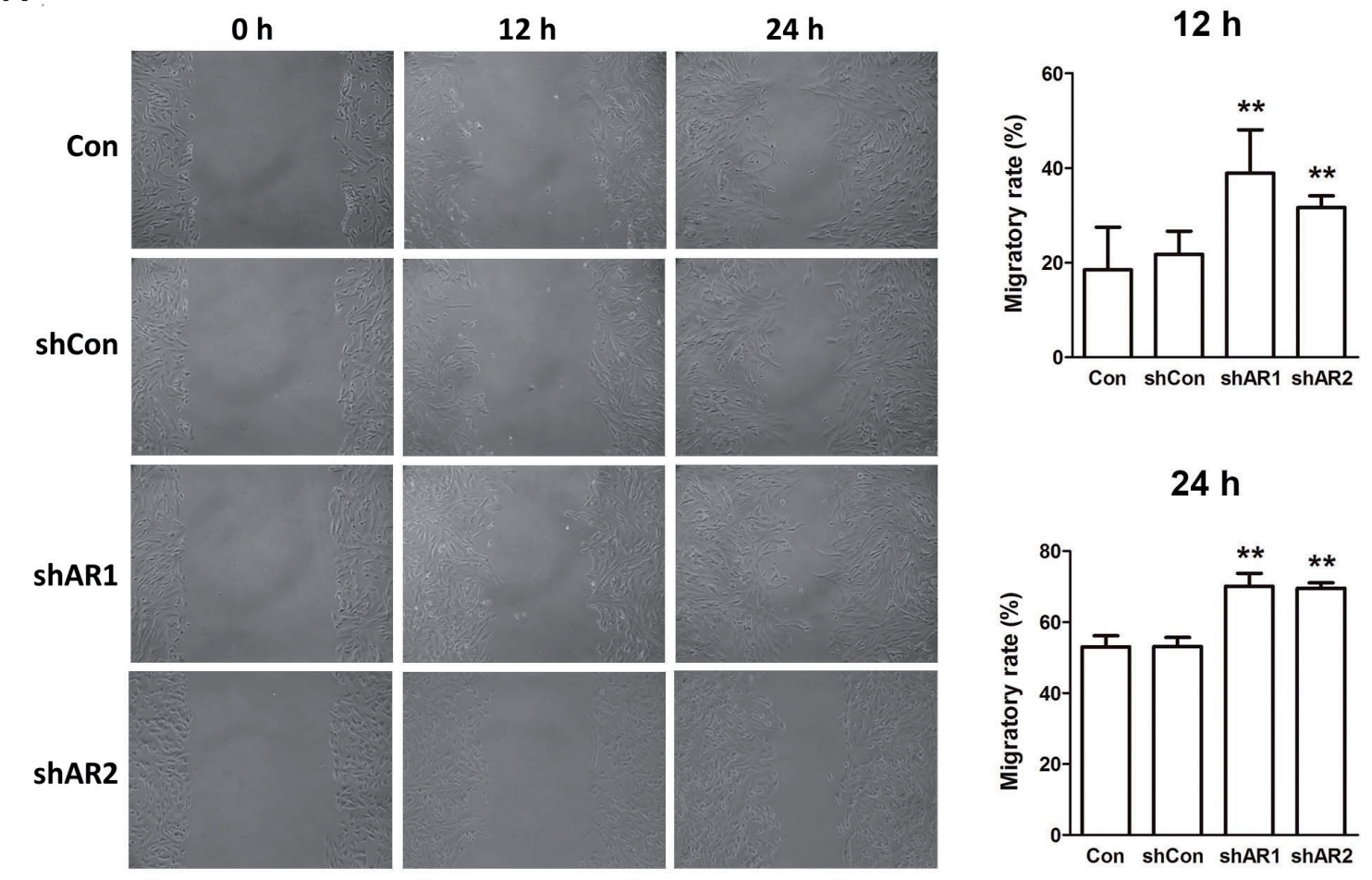

B
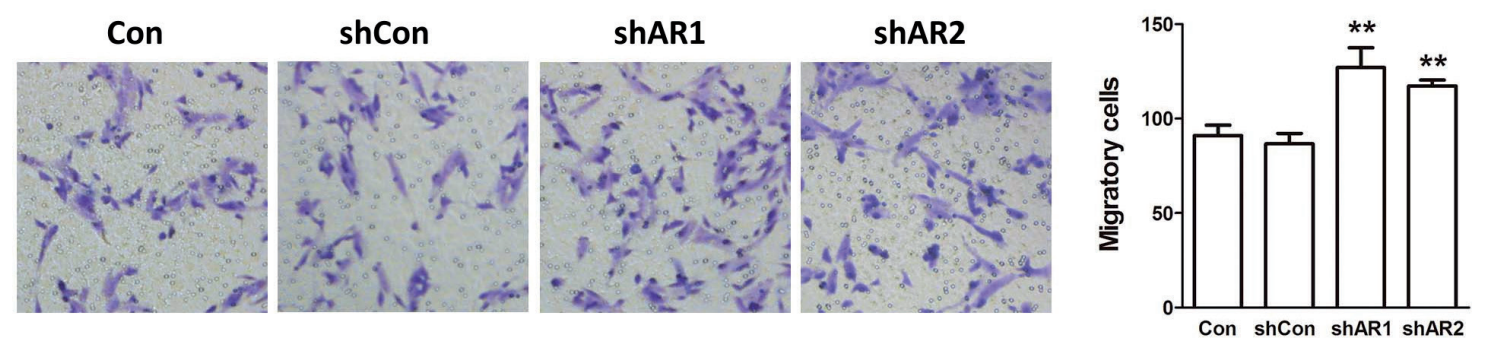

Figure 4. Knockdown of ARID1A promotes migration and invasion of SK-N-SH cells. The ability of migration and invasion was examined by wound healing (A) and Transwell assay (B). ARID1A knockdown significantly enhanced the ability of migration and invasion of SK-N-SH cells. Values are expressed as mean \pm standard deviation $(n=3) .{ }^{* *} P<0.01$ vs. shCon. Con: Control SK-N-SH cells; shCon: SK-N-SH cells transfected with scramble shRNA; shAR: SK-N-SH cells transfected with shRNA against ARID1A. 
reach significant. Meanwhile, the cell percentage of $S$ phase was markedly increased ( $P<0.01$ vs. shAR). However, the cell percentage of G2/M remained unchanged, which may because of the time point of examination. Therefore, we examined the cell cycle at $48 \mathrm{~h}$, both of the two shARID1A significantly reduced the cell percentage in G0/G1 phase $(P<0.01$ vs. shCon, Figure 3B). Meanwhile, the cells in the $\mathrm{S}$ and $\mathrm{G} 2 / \mathrm{M}$ phases were markedly increased. These data show that ARID1A knockdown causes G1 arrest inhibition and increases in DNA synthesis and mitosis in SK-N-SH cells.

Knockdown of ARID1A promotes migration and invasion of SK-N-SH cells. The migration ability of SK-N-SH cells was examined by wound healing assay. We found that migration rate was dramatically augmented when ARID1A gene was silenced in SK-N-SH cells compared with that of the scramble shRNA transfected cells $(P<0.01$, Figure 4 A). In addition, the invasion ability of SK-N-SH cells was examined by transwell assay. The number of cells invading through the gel and across the membranes in SK-N-SH cells stable transfected with shRNA against ARID1A was significantly increased compared with that of cells in shCon group. These data indicate that the migration and invasion capability of SK-N-SH cells is dramatically promoted by ARID1A knockdown. Both of the two sequences of shRNA target to ARID1A showed obvious pro-tumor effects on SK-N-SH cells, which indicate the important role of ARID1A as a tumor suppressor. Therefore, we chose one of the shRNA (shARID1A1) to perform the following experiments.

Knockdown of ARID1A enhances MMP-9 and MMP-2 expression levels and activity in SK-N-SH cells. MMP-9 and MMP-2 are involved in extracellular matrix (ECM) regulation, and are important for regulating cancer cell metastasis $[11,12]$. Gelatin zymography and Western blot analysis were performed to analyze the effect of ARID1A knockdown on the protein expression levels and activity of MMP-9 and MMP-2. The results showed that knockdown of ARID1A significantly elevated the protein expression levels and activity of MMP-9 and MMP-2 in SK-N-SH cells (Figure 5). These data suggest that MMP-9 and MMP-2 may be involved in the pro-tumorigenic effects of ARID1A knockdown in SK-N-SH cells.
A
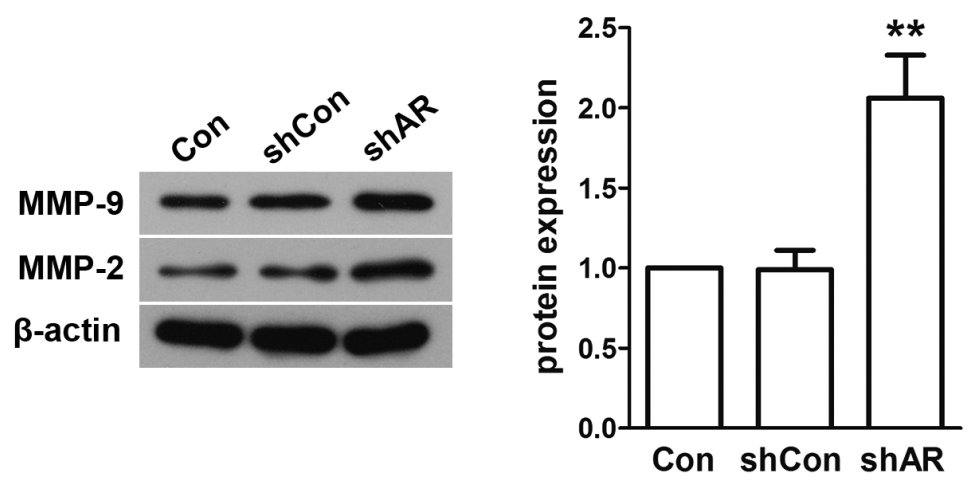

B

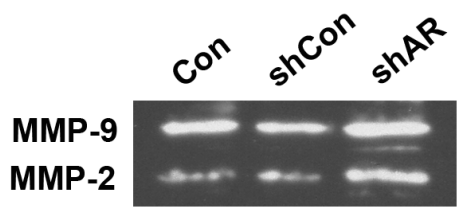

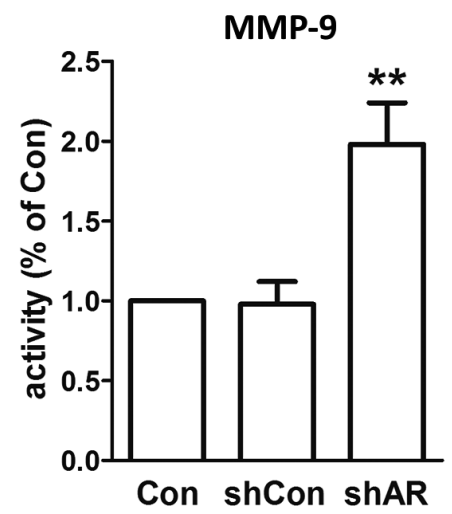

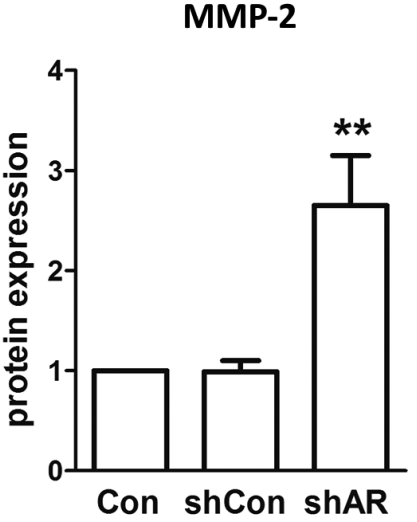

MMP-2

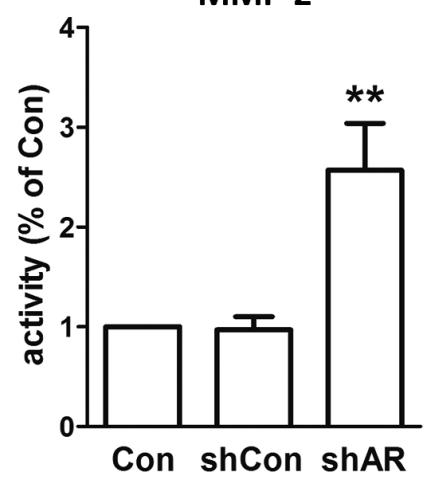

Figure 5. Knockdown of ARID1A enhances MMP-9 and MMP-2 expression levels and activity in SK-N-SH cells. (A) ARID1A knockdown upregulated protein expression of MMP-9 and MMP-2. (B) ARID1A knockdown increased MMP-9 and MMP-2 activity. Values are expressed as mean \pm standard deviation $(n=3) .{ }^{*} P<0.01$ vs. shCon. Con: Control SK-N-SH cells; shCon: SK-N-SH cells transfected with scramble shRNA; shAR: SK-N-SH cells transfected with shRNA against ARID1A. 
Knockdown of ARID1A induces EMT-like changes and nuclear translocation of $\beta$-catenin in SK-N-SH cells. Epithelial-to-mesenchymal transition (EMT) contributes to invasion and metastasis of tumor. In agreement with the previous study [13], SK-N-SH cells with ARID1A knockdown caused upregulation of epithelial marker E-cadherin and downregulation of mesenchymal marker $\mathrm{N}$-cadherin (Figure 6 ). Wnt/ $\beta$-catenin signaling plays essential role in regulation of EMT and metastasis [14]. Beta-catenin nuclear translocation was examined by Western blotting and immunofluorescence staining. From the Western blotting analysis, the protein expression of $\beta$-catenin was increased in the nuclei and decreased in the cytoplasm of the cells (Figure 7A). Immunofluorescence staining showed that $\beta$-catenin translocated into the nuclei of SK-N-SH cells after transfected with shRNA against ARID1A (Figure 7B). These observations indicate that ARID1A knockdown may induce EMT in SK-N-SH cells.

\section{Discussion}

In the present study, we found that shRNA-mediated ARID1A knockdown significantly increased cell proliferation, migration and invasion abilities in human neuroblastoma cell line SK-N-SH in vitro. The expression and activity of MMP-2 and 9 was enhanced after ARID1A knockdown. In addition, ARID1A knockdown induced EMT in SK-N-SH cells.
A.

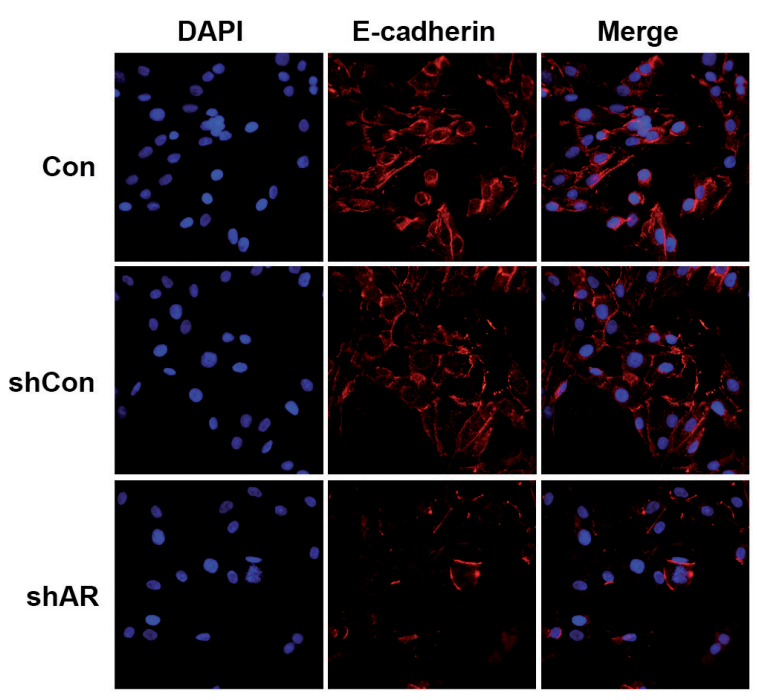

B

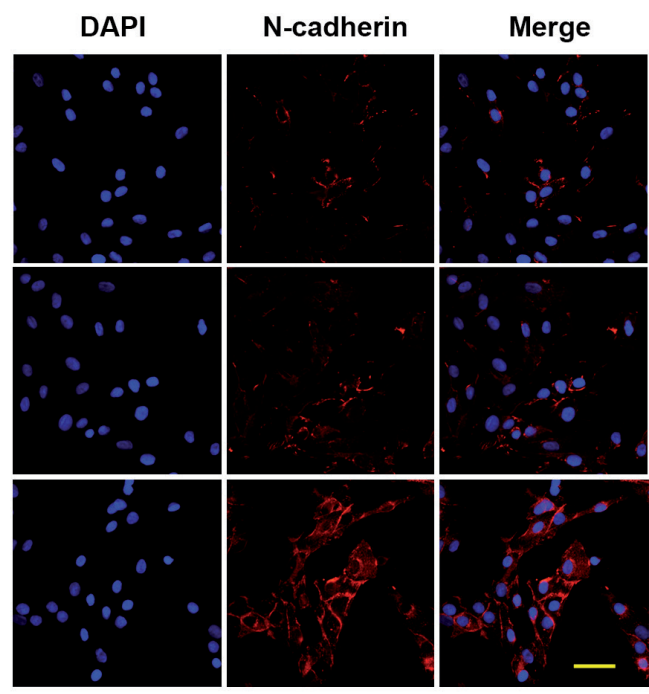

C

E-cadherin

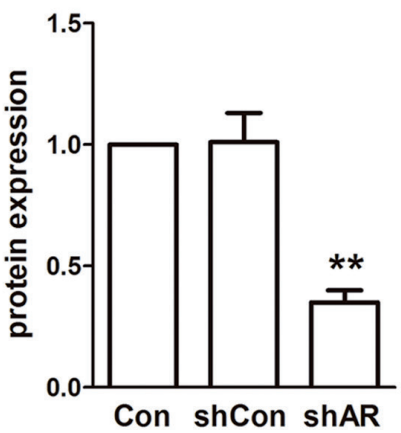

$\mathrm{N}$-cadherin

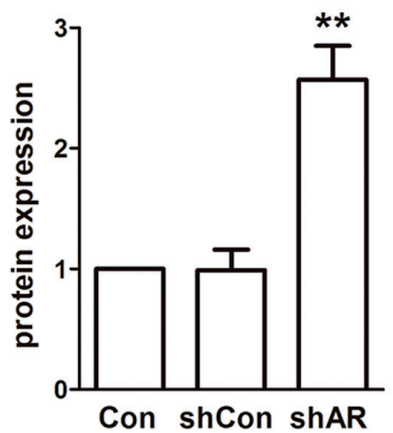

Figure 6. Knockdown of ARID1A induces EMT-like changes in SK-N-SH cells. ARID1A knockdown induced morphological change from epithelial to fibroblastic phenotype (A). In addition, examined by immunofluorescence staining (B) and Western blotting analysis (C), protein expression of E-cadherin was decreased and that of $\mathrm{N}$-cadherin and vimentin was increased. Scale bar: $\mathbf{5 0} \mu \mathrm{m}$. Values are expressed as mean \pm standard deviation $(n=3) .{ }^{* *} P<0.01$ vs. shCon. Con: Control SK-N-SH cells; shCon: SK-N-SH cells transfected with scramble shRNA; shAR: SK-N-SH cells transfected with shRNA against ARID1A. 
Cancer is a disease of uncontrolled cell growth and migration. The uncontrollable proliferation of tumor cells is usually due to the inappropriate regulation of cell cycle $[15,16]$. In the present study, RNAi was used to knock down the mRNA expression of ARID1A in SK-N-SH cells and the cellular viability, colony formation ability and cell cycle were examined. Results demonstrated that ARID1A knockdown increased cell growth and colony formation ability in SK-N-SH cells in comparison with control cells. A decrease of cell population at G0/G1 phase and an increase of that at S phase in ARID1A knockdown cells were also noted, which indicated the deregulation of cell cycle after ARID1A knockdown.

Metastasis is a hallmark of cancer that transforms the local tumor into a systemic disease and is the primary causes of mortality in cancer patients [17]. Migration and invasion of cancer cells is the major step of metastasis. In the present study, wound healing and Transwell assays were performed to determine the effects of ARID1A knockdown on the migration and invasion abilities of SK-N-SH cells. The data indicated that knockdown of ARID1A significantly enhanced the migration and invasion ability of the SK-N-SH cells.

Metastasis of malignant cancer cells is a process that contains a series steps including cell-cell connections losing, cells releasing, extracellular matrix (ECM) degradation, and cell penetration into circulatory system [18]. MMPs, a family of endopeptidases whose enzymatic activity is determined by $\mathrm{Zn}^{2+}$ ions, play crucial roles in proteolytic degradation of ECM, thereby facilitating cancer cells invasion and metastasis. In particular, MMP-2 and 9 have been considered to contribute to various cancers and have been identified as cancer markers [19]. Overexpression of these two members of MMPs family have been found to contribute to the increase of invasion ability in human neuroblastoma cell lines, and MMP-2 and 9 expression levels were associated with the ability of invasion in neuroblastoma cells [20-23]. In the present study, the protein expression and activity of MMP-2 and 9 were increased in SK-N-SH cells with ARID1A knockdown. This finding is agreement with the previous study on ovarian clear cell carcinoma ES2 cell line [24], and indicates that MMPs regulation may be involved in the tumor suppression effects of ARID1A.

Cadherins are a family of transmembrane glycoproteins that mediate calcium-dependent cell adhesion and play important roles in maintenance of tissue epithelial structure. E-cadherin and $\mathrm{N}$-cadherin are two widely studied cadherins in cancer. E-cadherin is involved in maintenance of the normal phenotype of epithelial cells $[25,26]$. Expression of endogenous E-cadherin suppressed cellular motility in rat astrocyte-like WC5 cells [27]. However, unlike E-cadherin, N-cadherin promotes motility and invasion in malignant cancer cells [28]. $\mathrm{N}$-cadherin was found overexpression in various malignant cancer cells, and $\mathrm{N}$-cadherin expression promote invasion in
A
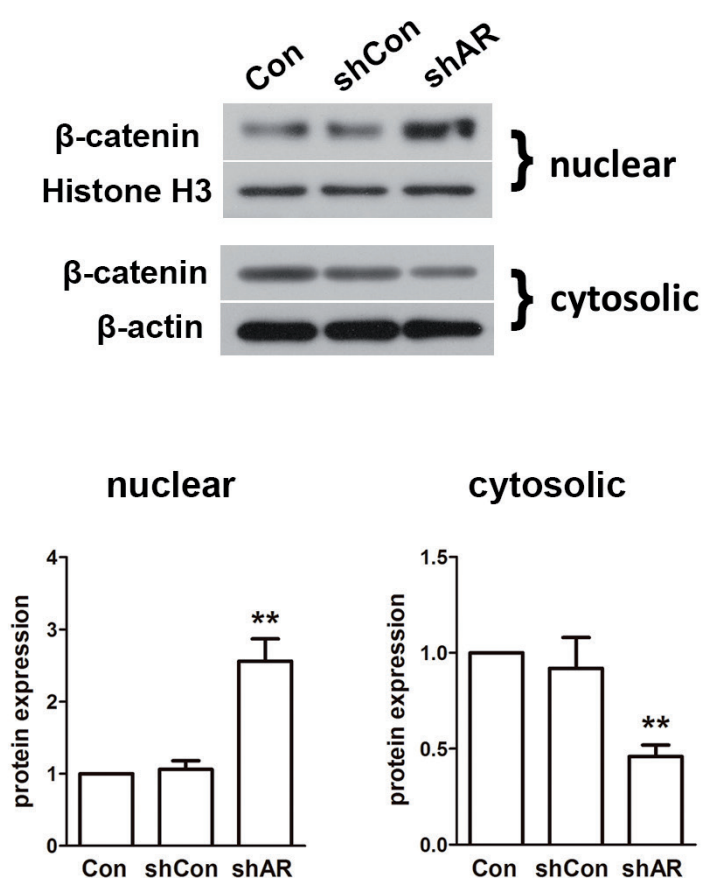

B
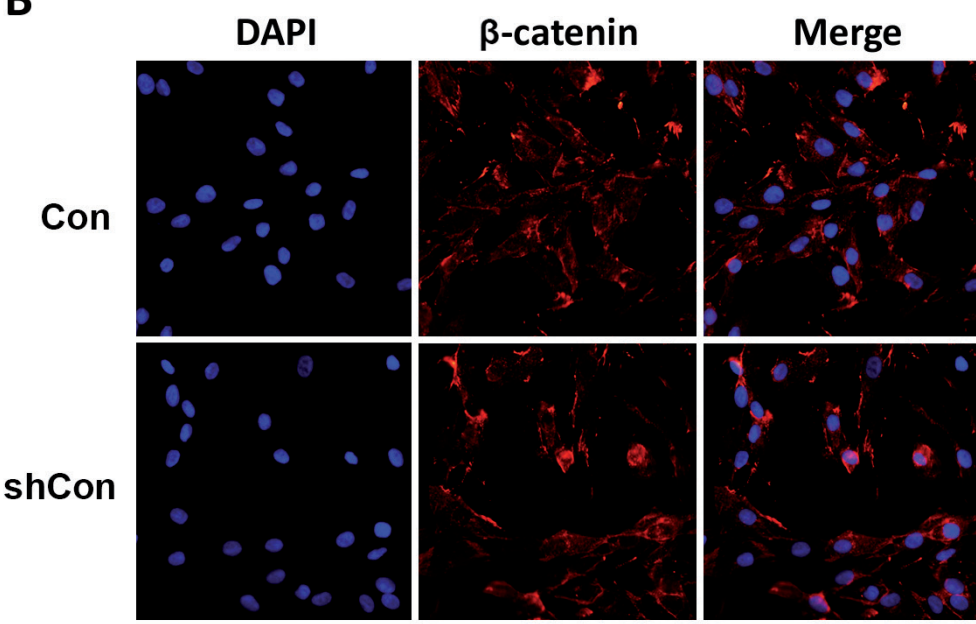

ShAR
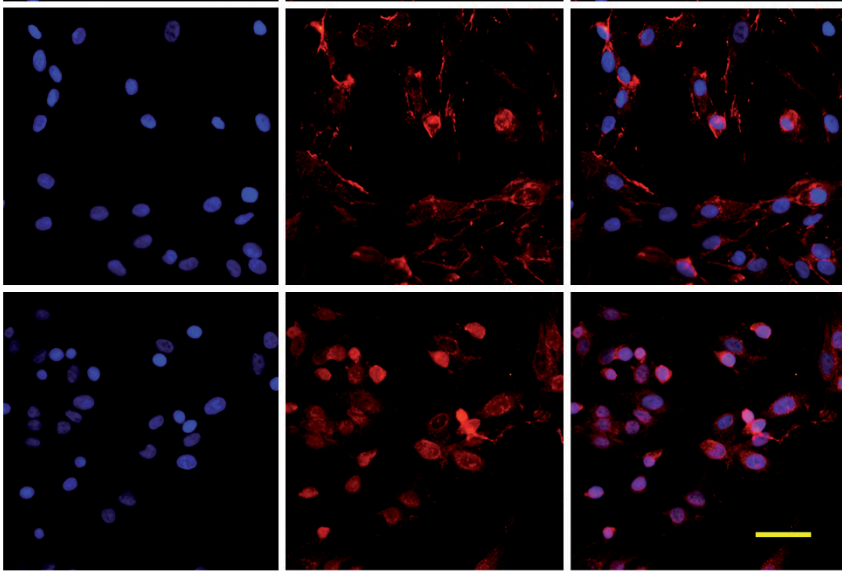

Figure 7. Knockdown of ARID1A induces nuclear translocation of $\beta$-catenin in SK-N-SH cells. ARID1A knockdown induced increased protein expression of $\beta$-catenin in the nuclei and decreased expression in the cytoplasm in SK-N-SH cells (A). Immunofluorescence staining showed the nuclear translocation of $\beta$-catenin in SK-N-SH cells. (B) Scale bar: $50 \mu \mathrm{m}$. Values are expressed as mean \pm standard deviation ( $\mathrm{n}=3$ ). ${ }^{\star \star} \boldsymbol{P}<\mathbf{0 . 0 1}$ vs. shCon. Con: Control SK-N-SH cells; shCon: SK-N-SH cells transfected with scramble shRNA; shAR: SK-N-SH cells transfected with shRNA against ARID1A. 
cancer cells regardless the expression of E-cadherin [29, 30]. In previous study, Yan et al found that ARID1A silencing downregulated E-cadherin expression, and ARID1A overexpression upregulated E-cadherin expression in gastric cancer cells [13]. In agreement with this finding, we found that the expression of E-cadherin was enhanced and that of $\mathrm{N}$-cadherin was diminished in ARID1A knockdown SN-K-SH cells. This switch from $\mathrm{E}$-cadherin expression to $\mathrm{N}$-cadherin expression indicates the $\mathrm{SN}-\mathrm{K}-\mathrm{SH}$ cells become more invasive.

$\mathrm{E}$-cadherin and $\mathrm{N}$-cadherin were also considered as the EMT markers. EMT is identified as epithelial cells lose their epithelial phenotype and gain mesenchymal characters and is involved in metastasis in epithelial cancers [31]. Neuroblastoma cells may also undergo EMT-like phenotype in the process of metastasis although neuroblastoma is of neuroectodermal origin $[32,33]$. We also noted that $\beta$-catenin nuclear translocation was increased after ARID1A knockdown. Deregulation of $\mathrm{Wnt} / \beta$-catenin signaling is involved in the development of many diseases including cancer [34]. Beta-catenin regulates EMT-associated genes through $\beta$-catenin/TCF signaling. During EMT, $\beta$-catenin translocates to nuclear and activates the downstream target gene like snail, and the latter induces EMT through inhibiting epithelial markers including E-cadherin [35-37]. The EMT-like changes we found in SK-K-SH cells indicated that ARID1A knockdown enhanced migration and invasion abilities via altering EMT-associated proteins.

In summary, shRNA-mediated ARID1A knockdown increased the activity and expression of MMP-2 and 9, induces E-cadherin downregulation, $\mathrm{N}$-cadherin upregulation and nuclear translocation of $\beta$-catenin, enhancing SK-K-SH cells migration and invasion. Our findings suggest ARID1A is a tumor suppressor in neuroblastoma.

Acknowledgements: This study was supported by a grant from the National Natural Science Foundation of China (No. 81400438).

\section{References}

[1] MARIS JM. Recent advances in neuroblastoma. N Engl J Med 2010; 362: 2202-2211. https://doi.org/10.1056/NEJM$\underline{\mathrm{ra} 0804577}$

[2] MARIS JM, HOGARTY MD, BAGATELL R, COHN SL. Neuroblastoma. Lancet 2007; 369: 2106-2120. https://doi. org/10.1016/S0140-6736(07)60983-0

[3] MULLASSERY D, DOMINICI C, JESUDASON EC, MCDOWELL HP, LOSTY PD. Neuroblastoma: contemporary management. Arch Dis Child Educ Pract Ed 2009; 94: 177-185. https://doi.org/10.1136/adc.2008.143909

[4] MODAK S, CHEUNG NK. Neuroblastoma: Therapeutic strategies for a clinical enigma. Cancer Treat Rev 2010; 36: 307-317. https://doi.org/10.1016/j.ctrv.2010.02.006

[5] HARGREAVES DC, CRABTREE GR. ATP-dependent chromatin remodeling: genetics, genomics and mechanisms. Cell Res 2011; 21: 396-420. https://doi.org/10.1038/cr.2011.32
[6] DAWSON MA, KOUZARIDES T. Cancer epigenetics: from mechanism to therapy. Cell 2012; 150: 12-27. https://doi. org/10.1016/j.cell.2012.06.013

[7] WU JN, ROBERTS CW. ARID1A mutations in cancer: another epigenetic tumor suppressor? Cancer Discov 2013; 3: 35-43. https://doi.org/10.1158/2159-8290.CD-12-0361

[8] AYHAN A, MAO TL, SECKIN T, WU CH, GUAN B et al. Loss of ARID1A expression is an early molecular event in tumor progression from ovarian endometriotic cyst to clear cell and endometrioid carcinoma. Int J Gynecol Cancer 2012; 22: 1310-1315. https://doi.org/10.1097/IGC.0b013e31826b5dcc

[9] GUAN B, MAO TL, PANUGANTI PK, KUHN E, KURMAN RJ et al. Mutation and loss of expression of ARID1A in uterine low-grade endometrioid carcinoma. Am J Surg Pathol 2011; 35: 625-632. https://doi.org/10.1097/PAS.0b013e318212782a

[10] SAUSEN M, LEARY RJ, JONES S, WU J, REYNOLDS CP et al. Integrated genomic analyses identify ARID1A and ARID1B alterations in the childhood cancer neuroblastoma. Nat Genet 2013; 45: 12-17. https://doi.org/10.1038/ng.2493

[11] ULRICH R, GERHAUSER I, SEELIGER F, BAUMGARTNER W, ALLDINGER S. Matrix metalloproteinases and their inhibitors in the developing mouse brain and spinal cord: a reverse transcription quantitative polymerase chain reaction study. Dev Neurosci 2005; 27: 408-418. https://doi. org/10.1159/000088455

[12] ZHAO BQ, WANG S, KIM HY, STORRIE H, ROSEN BR et al. Role of matrix metalloproteinases in delayed cortical responses after stroke. Nat Med 2006; 12: 441-445. https:// doi.org/10.1038/nm1387

[13] YAN HB, WANG XF, ZHANG Q, TANG ZQ, JIANG YH et al. Reduced expression of the chromatin remodeling gene ARID1A enhances gastric cancer cell migration and invasion via downregulation of E-cadherin transcription. Carcinogenesis 2014; 35: 867-876. https://doi.org/10.1093/carcin/bgt398

[14] MACDONALD BT, TAMAI K, HE X. Wnt/beta-catenin signaling: components, mechanisms, and diseases. Dev Cell 2009; 17: 9-26. https://doi.org/10.1016/j.devcel.2009.06.016

[15] NAULT JC, ZUCMAN-ROSSI J. TERT promoter mutations in primary liver tumors. Clin Res Hepatol Gastroenterol 2016; 40: 9-14. https://doi.org/10.1016/j.clinre.2015.07.006

[16] MALUMBRES M, BARBACID M. Cell cycle, CDKs and cancer: a changing paradigm. Nat Rev Cancer 2009; 9: 153-166. https://doi.org/10.1038/nrc2602

[17] HANAHAN D, WEINBERG RA. Hallmarks of cancer: the next generation. Cell 2011; 144: 646-674. https://doi. org/10.1016/j.cell.2011.02.013

[18] YOON SO, PARK SJ, YUN CH, CHUNG AS. Roles of matrix metalloproteinases in tumor metastasis and angiogenesis. J Biochem Mol Biol 2003; 36: 128-137. https://doi.org/10.5483/ bmbrep.2003.36.1.128

[19] BAUVOIS B. New facets of matrix metalloproteinases MMP-2 and MMP-9 as cell surface transducers: outside-in signaling and relationship to tumor progression. Biochim Biophys Acta 2012; 1825: 29-36. https://doi.org/10.1016/j. bbcan.2011.10.001

[20] LU HF, LAI KC, HSU SC, LIN HJ, KUO CL et al. Involvement of matrix metalloproteinases on the inhibition of cells 
invasion and migration by emodin in human neuroblastoma SH-SY5Y cells. Neurochem Res 2009; 34: 1575-1583. https:// doi.org/10.1007/s11064-009-9946-3

[21] LEE HJ, PARK MK, BAE HC, YOON HJ, KIM SY et al. Transglutaminase-2 Is Involved in All-Trans Retinoic Acid-Induced Invasion and Matrix Metalloproteinases Expression of $\mathrm{SH}$ SY5Y Neuroblastoma Cells via NF-kappaB Pathway. Biomol Ther (Seoul) 2012; 20: 286-292. https://doi.org/10.4062/ biomolther.2012.20.3.286

[22] MOHAN N, AI W, CHAKRABARTI M, BANIK NL, RAY SK. KLF4 overexpression and apigenin treatment down regulated anti-apoptotic $\mathrm{Bcl}-2$ proteins and matrix metalloproteinases to control growth of human malignant neuroblastoma SK-N-DZ and IMR-32 cells. Mol Oncol 2013; 7: 464-474. https://doi. org/10.1016/j.molonc.2012.12.002

[23] BJORNLAND K, BRATLAND A, RUGNES E, PETTERSEN $S$, JOHANSEN HT et al. Expression of matrix metalloproteinases and the metastasis-associated gene S100A4 in human neuroblastoma and primitive neuroectodermal tumor cells. J Pediatr Surg 2001; 36: 1040-1044. https://doi.org/10.1053/ jpsu.2001.24735

[24] LYU CS, ZHANG YL, LANG JH. Impact of biological function on ovarian clear cell carcinoma ES2 cell line with ARID1A gene expression down-regulating in vitro. Zhonghua Fu Chan Ke Za Zhi 2016; 51: 209-215.

[25] BEHRENS J. Cadherins and catenins: role in signal transduction and tumor progression. Cancer Metastasis Rev 1999; 18: 15-30. https://doi.org/10.1023/A:1006200102166

[26] EL-BAHRAWY MA, PIGNATELli M. E-cadherin and catenins: molecules with versatile roles in normal and neoplastic epithelial cell biology. Microsc Res Tech 1998; 43: 224-232. https://doi.org/10.1002/(SICI)10970029(19981101)43:3<224::AID-JEMT4>3.0.CO;2-Q

[27] CHEN H, PARADIES NE, FEDOR-CHAIKEN M, BRACKENBURY R. E-cadherin mediates adhesion and suppresses cell motility via distinct mechanisms. J Cell Sci 1997; 110 (Pt 3): 345-356.

[28] KIM J B, ISLAM S, KIM YJ, PRUDOFF RS, SASS KM et al. N-Cadherin extracellular repeat 4 mediates epithelial to mesenchymal transition and increased motility. J Cell Biol 2000; 151: 1193-1206. https://doi.org/10.1083/ jcb.151.6.1193

[29] NIEMAN MT, PRUDOFF RS, JOHNSON KR, WHEELOCK MJ. N-cadherin promotes motility in human breast cancer cells regardless of their E-cadherin expression. J Cell Biol 1999; 147: 631-644. https://doi.org/10.1083/jcb.147.3.631

[30] SHAMIR ER, EWALD AJ. Adhesion in mammary development: novel roles for E-cadherin in individual and collective cell migration. Curr Top Dev Biol 2015; 112: 353-382. https:// doi.org/10.1016/bs.ctdb.2014.12.001

[31] THIERY JP, ACLOQUE H, HUANG RY, NIETO MA. Epithelial-mesenchymal transitions in development and disease. Cell 2009; 139: 871-890. https://doi.org/10.1016/j. cell.2009.11.007

[32] VITALI R, MANCINI C, CESI V, TANNO B, MANCUSO $\mathrm{M}$ et al. Slug (SNAI2) down-regulation by RNA interference facilitates apoptosis and inhibits invasive growth in neuroblastoma preclinical models. Clin Cancer Res 2008; 14: 4622-4630. https://doi.org/10.1158/1078-0432.CCR-07-5210

[33] KIM NH, KIM HS, LI XY, LEE I, CHOI HS et al. A p53/ miRNA-34 axis regulates Snail1-dependent cancer cell epithelial-mesenchymal transition. J Cell Biol 2011; 195: 417-433. https://doi.org/10.1083/jcb.201103097

[34] MOON RT, KOHN AD, DE FERRARI GV, KAYKAS A. WNT and beta-catenin signalling: diseases and therapies. Nat Rev Genet 2004; 5: 691-701. https://doi.org/10.1038/nrg1427

[35] MA X, YAN W, DAI Z, GAO X, MA Y et al. Baicalein suppresses metastasis of breast cancer cells by inhibiting EMT via downregulation of SATB1 and Wnt/beta-catenin pathway. Drug Des Devel Ther 2016; 10: 1419-1441. https://doi. org/10.2147/DDDT.S102541

[36] BIENZ M. beta-Catenin: a pivot between cell adhesion and Wnt signalling. Curr Biol 2005; 15: R64-67. https://doi. org/10.1016/j.cub.2004.12.058

[37] YOOK JI, LI XY, OTA I, HU C, KIM HS et al. A WntAxin2-GSK3beta cascade regulates Snaill activity in breast cancer cells. Nat Cell Biol 2006; 8: 1398-1406. https://doi. org/10.1038/ncb 1508 